\title{
Potential glucose monitoring of blood plasma using hollow core photonic crystal fibre
}

Horan, L., Khara, G., Rutowska, M., Ellis, A., Garcia Gunning, F.

L. E. Horan, G. Khara, M. Rutowska, A. D Ellis, F. C Garcia Gunning, "Potential glucose monitoring of blood plasma using hollow core photonic crystal fibre," Proc. SPIE 7753, 21st International Conference on Optical Fiber Sensors, 77533W (17 May 2011); doi: 10.1117/12.885005

SPIE Event: 21st International Conference on Optical Fibre Sensors (OFS21), 2011, Ottawa, Canada 


\title{
Potential Glucose Monitoring of Blood Plasma Using Hollow Core Photonic Crystal Fibre
}

\author{
L. E. Horan, G. Khara, M. Rutowska, A. D. Ellis, F. C. Garcia Gunning \\ Tyndall National Institute and Department of Physics, University College Cork, Ireland \\ laura.horan@tyndall.ie
}

\begin{abstract}
The ratio $(\xi)$ of surface tension to viscosity of liquids can be determined using hollow core photonic crystal fibres (HCPCF), and we show here techniques to determine $\xi$ of glucose levels within fluids, of nano-litre quantities. We demonstrate an optically integrated micro-capillary viscometer, to determine the concentrations of nano-litre solutions based on properties of their flow within HC-PCF. The filling of the fibres with liquids within a given range of refractive index will induce a shift in the photonic band gap of the fibre, allowing guidance of light at wavelengths that were originally outside the bandgap of the HC-PCF.
\end{abstract}

Keywords: hollow core photonic crystal fibre, glucose, bio-sensing, biological fluids.

\section{INTRODUCTION}

Managing diabetes and glucose levels within the blood is an ongoing challenge. The human body naturally releases insulin to maintain the whole blood glucose level below $7.6 \mathrm{mmol} / \mathrm{L}$ after the ingestion of food, and is usually much lower during fasting, with normal ranges varying between $4-6 \mathrm{mmol} / \mathrm{L}^{[1]}$. In patients with diabetes, insulin release is impeded, causing possible nerve ending damage, cardiovascular disease, kidney failure, and due to complications from diabetes, limb amputation, stroke and death. The International Organisation of Standards specifies that commercially available point of care glucose monitors need to have an accuracy that is within $20 \%$ of lab diagnosis results ${ }^{[2]}$, for patients monitoring glucose levels.

The properties of a photonic crystal fibres (PCF) have revolutionised optical fibre sensing, because of their structure and light guiding properties ${ }^{[3]}$. PCF are optical waveguides that consist of a periodic microstructure of hollow capillaries, and allow the guidance of light by the photonic band gap (PBG) effect ${ }^{[4]}$, where all light is either reflected towards or away (scattered) from the core. PCFs have been applied to the areas of gas, temperature and bio sensing ${ }^{[5-7]}$. These fibres are appealing for use as they allow the insertion of liquid or gas samples into the core and cladding holes, enabling a large overlap between the samples and optical field, when compared with tapered standard optical fibres employing the evanescent field for sensing ${ }^{[8]}$.

In this paper we demonstrate the use of a hollow core PCF (HC-PCF) viscometer for the detection of concentrations of glucose dissolved in distilled water, suggesting that HC-PCF could be used for the monitoring of glucose levels within blood plasma. The PBG shift and changing light guiding properties, along with the dominance of surface tension at the micro scale are utilised to determine the ratio of surface tension to viscosity $(\xi)$ of glucose solutions via a micro-capillary viscometer set-up. To the best of our knowledge, liquid viscosity $(\mu)$ and surface tension $(\sigma)$ ratio measurements using nano-litre samples in a HC-PCF have not been reported before. In contrast to our methods, current optical viscometers utilise methods that determine the refractive index of a liquid, rather than measuring the physical parameters of surface tension and viscosity. Refractive index values depend and vary on sample and liquid concentration ${ }^{[9]}$. Current nano-liter viscometers require a complex structure of channels and analysis for viscosities measurement. Others require a constant monitoring of the liquid air interface via CCD camera as the liquid flows through micro-channels via capillary action ${ }^{[10]}$.

\section{PRINCIPLES OF MEASUREMENT}

The HC-PCFs used for the purposes of this experiment are commercially available HC-1060 (NKT Photonics A/S) as shown in figure 1(a). The core of the HC-PCF is $10 \mu \mathrm{m}$ in diameter, and surface tension, resisted by the fluids viscosity is

21st International Conference on Optical Fiber Sensors, edited by Wojtek J. Bock, Jacques Albert, Xiaoyi Bao,

Proc. of SPIE Vol. 7753, 77533W · @ 2011 SPIE · CCC code: 0277-786X/11/\$18 · doi: 10.1117/12.885005 
the dominant force in the flow of liquid through the capillaries. The flow of liquid is monitored to measure $\xi$ of the nano litre quantities of liquids. This allows the micro capillary viscometer to pick up minute changes in the concentration of glucose within the sample, due to the strong reliance of the viscosity of the sample on its surface tension and temperature.

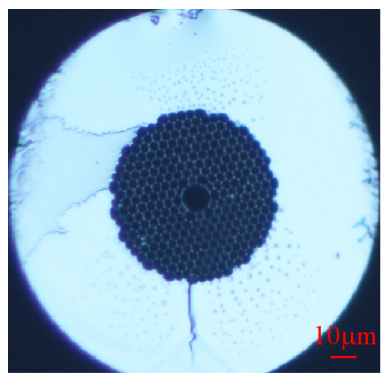

(a)

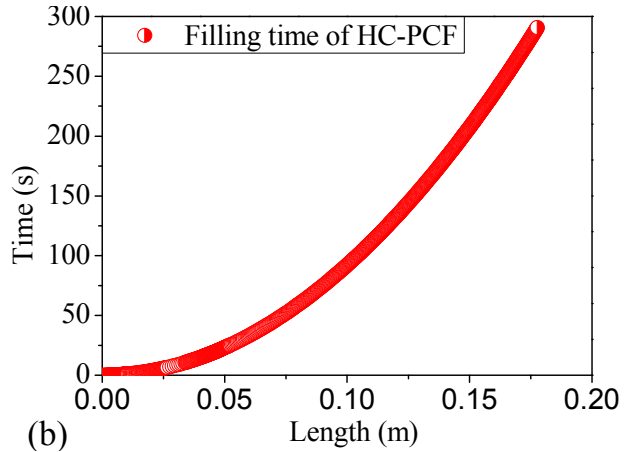

(b)

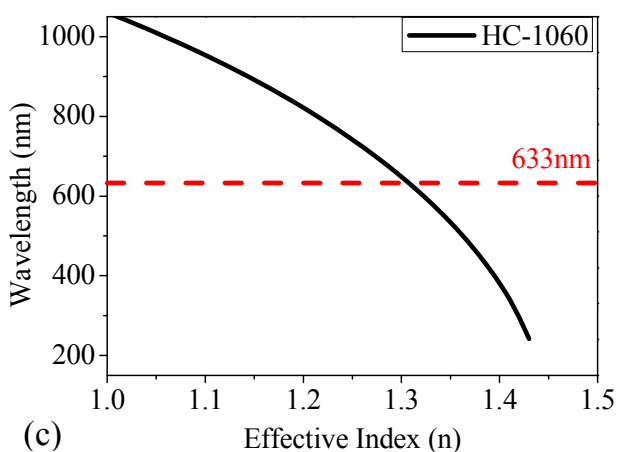

(c)

Figure 1: (a) Cross section of HC-PCF, type HC-1060 used in experiments, viewed under microscope (x50). (b)The theoretical prediction for the time taken to fill a length of the core capillary HC-PCF, HC-1060 with diameter $10 \mu \mathrm{m}$. (c) The theoretically predicted band gap shift for HC-1060, showing the effective index of the microstructure, and guidance for HeNe laser 633nm.

When one end of a HC-PCF is immersed in a fluid sample, the hollow capillaries will fill, with the core filling more rapidly than the capillaries in the cladding. The flow of liquid through the HC-PCF is well known ${ }^{[11]}$, and results in a filling time $(t)$ dependent on capillary radius $(r)$, fibre length $(L)$ and the ratio of surface tension to viscosity. Considering the forces acting on a column of liquid the ratio $\xi$ of surface tension to viscosity may be calculated, as presented in equation (1). A theoretical filling characteristic for HC-1060 is shown in figure 1(b). This quadratic characteristic may be used to approximate the minimum required fibre length for a given time measurement.

$$
\xi=\frac{\sigma}{\mu}=\frac{2 L^{2}}{t r}
$$

Note that with pure water we have previously reported that repeatable results are only possible after the HC-PCF has been filled with water several times ${ }^{[12]}$. This was attributed to the hydrophobic quality of the glass surface, which required the application of a thin film coating (wetting) for unrestricted movement of water through the capillaries ${ }^{[11]}$.

The introduction of the liquid to the hollow capillaries of HC-PCF modifies the PBG effect. When filled, the allowed wavelength is shifted ${ }^{[13]}$, as shown in figure 1(c). The filling time can be estimated from the transmission of light at the shifted PBG wavelength. Different glucose concentrations will have different refractive indices, viscosities and surface tensions. $\xi$ may be determined directly from the filling time, whilst the index may be determined by the PBG shift.

\section{GLUCOSE EXPERIMENT}

A $633 \mathrm{~nm}$ HeNe laser is guided into the core of a short length $(10-20 \mathrm{~cm})$ of the HC-PCF using infinity corrected lenses and a nano positioning stage. For an unfilled fibre, light is weakly guided through the entire microstructure with only a weak bandgap effect at $633 \mathrm{~nm}$. Light exiting the fibre is collimated and passed through a beam splitter to enable the output to be simultaneously observed using a photo detector and a CCD camera. A typical CCD image for an unfilled fibre is shown in the top left hand inset to figure 2(b), demonstrating that there is no bandgap guidance within the fibre core, and light is confined to the silica struts and hollow core. One end of the HC-PCF is held in a reservoir, which is kept at a constant temperature $\left( \pm 0.1^{\circ} \mathrm{C}\right)$ by a peltier heating element and temperature controller (TEC). Liquid is introduced to the reservoir and fills the HC-PCF as described in section 2.

Filling the HC-PCF with liquid changes the light guiding properties of the HC-PCF due to the changing effective index, and can be seen from the photodiode as a change in power, shown in figure 2(b). Recall that the initial empty fibre has limited guidance. When liquid is added to the reservoir, it scatters light, so a reduced portion will be detected at the 
photodiode. Between 25 and 260 seconds, the core will fill from the output end. This allows light to be guided by total internal reflection since the refractive index in the core is larger than that of the microstructure cladding. Once a significant fraction of the capillaries which comprise the cladding begin to fill, there will be a portion of the fibre that will be completely filled with liquid, allowing guidance of $633 \mathrm{~nm}$ light by the shifted PBG effect ${ }^{[14]}$. This gives a strongly guided signal as shown by the CCD image at 260 seconds in figure 2(b).

\section{$\mathrm{HeNe}$}

(a)

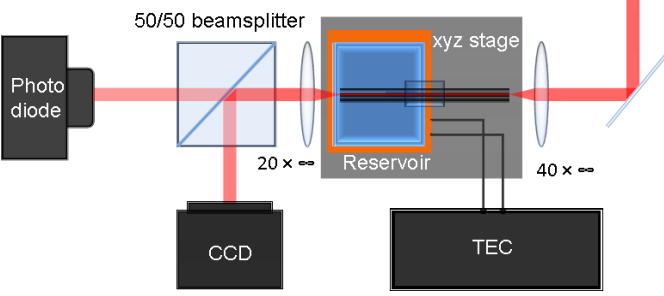

(b)

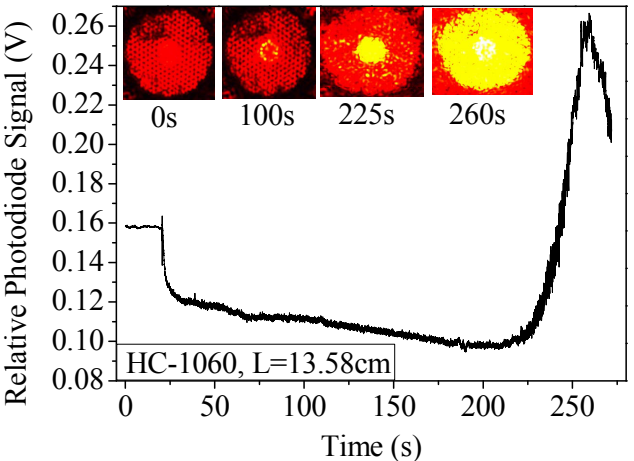

Figure 2: (a) The experimental set-up for the experiment, the fibre inserted into the temperature controlled reservoir (TEC), and output measured by the CCD and photodiode. 20xœ and 40xœ lenses are used to focus HeNe laser. (b)The shift in the PBG, seen as an increase in signal by the photodiode, which occurs as the fibre fills. The inserts correspond to the images captured by the CCD during the filling of the HC-PCF,

The performance of the system was characterised using a set of samples prepared from distilled water and anhydrous 96\% Sigma-Aldrich D-(+)-Glucose. These were combined to create glucose solutions of concentrations typically found in blood plasma and spanning the hypoglycemic and hyperglycemic regions ( 3 to $\left.9 \mathrm{mmol} / \mathrm{L}^{[1]}\right)$ Samples from each solution were introduced to various HC-PCF fibre lengths ranging from 10 to $20 \mathrm{~cm}$, where each fibre was used once.

\section{RESULTS}
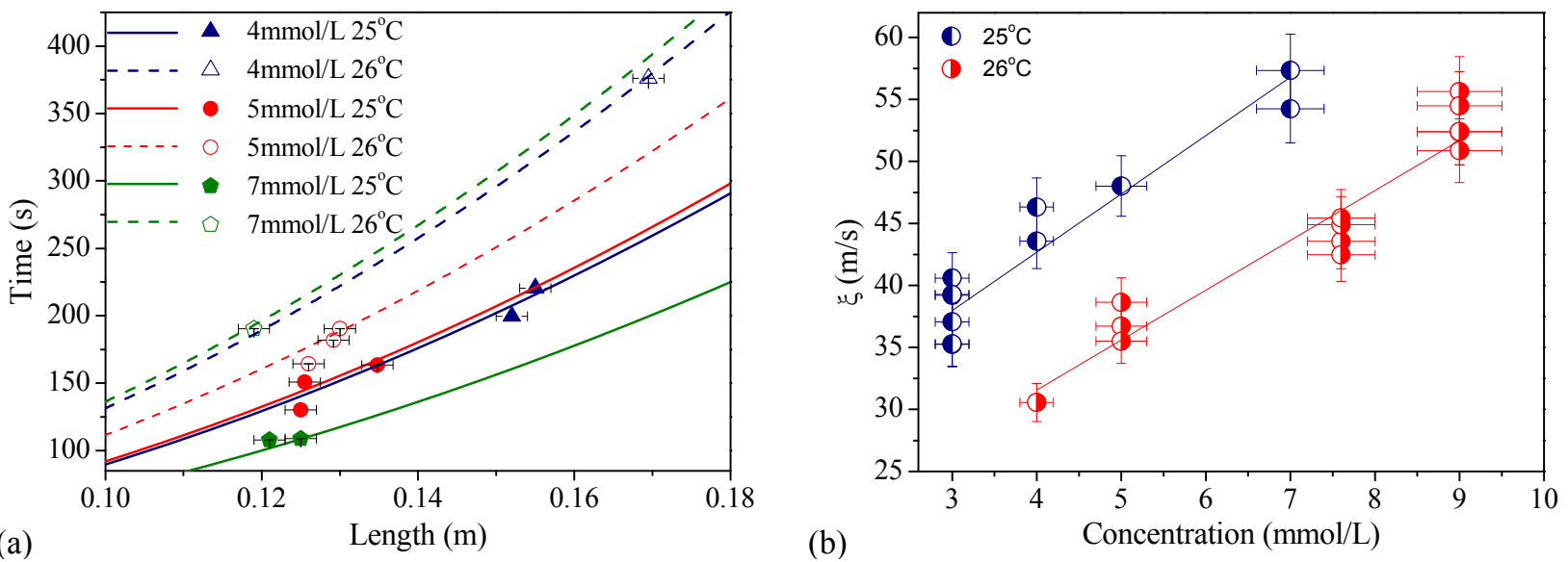

Figure 3: (a) Experimental data for batches measured with different temperatures plotted to theoretical function from $^{[11]}$. (b) Graph indicating the two batched of samples measured at $25^{\circ} \mathrm{C}$ and $26^{\circ} \mathrm{C}$.

From measurement results as shown in figure 3(a), for each concentration, the data follows the theoretical quadratic fit, as per $^{[11]}$. Note that for a given fibre, the filling time was highly reproducible, indicating that even such small concentrations of solute are effective in overcoming the filling variability associated with pure water. Figure 3(b) shows the directly measured results for the batches of samples, at temperatures $25^{\circ} \mathrm{C}$ and $26^{\circ} \mathrm{C}$. This clearly shows the expected 
linear variation in filling time with concentration, but also demonstrates the critical importance of accurate temperature control, with a $1{ }^{\circ} \mathrm{C}$ shift resulting in a change in slope of $10 \%$.

Up to five measurements were taken for each sample, each using a different fibre length. The sample standard deviation was $4.42 \mathrm{~m} / \mathrm{s}$, which, as can be seen from figure 3, can be primarily attributed to our length measurement, with an accuracy of $0.01 \mathrm{~cm}$. Simple improvements in the uniformity of HC-PCF lengths would greatly enhance the accuracy of this measurement technique to well within the $20 \%$ requirement for point of care diagnosis.

\section{CONCLUSION}

We have shown that HC-PCF can be used as a detector to determine the $\xi$ ratio of glucose solution samples, which can determine and identify glucose levels to be within the normal, hyper and hypoglycemic ranges. We anticipate that this form of analysis will have a wide range of uses in the analysis of biological fluids and chemical detection in pharmaceutical and medical industries, amongst others.

\section{ACKNOWLEDGEMENTS}

I would like to thank Jiadi Lu and Mariana Brandao for invaluable help. L. Horan would like to thank IRCSET Embark Initiative for a PhD fellowship. This work was supported by Science Foundation Ireland under grant number 6/IN/I969.

\section{REFERENCES}

[1] Polonsky, K. S., Given, B. D., and Van Cauter, E., "Twenty-four-hour profiles and pulsatile patterns of insulin secretion in normal and obese subjects," The J. of Clin. Inv., 81(2), 442-448 (1988).

[2] I. O. f. Standardisation, [ International Organisation for Standardisation: Requirements for In Vitro Blood Glucose Monitoring Systems for Self-Testing in Managing Diabetes Mellitus, Geneva(2001).

[3] Birks, T. A., Roberts, P. J., Russell, P. S. J. et al., "Full 2-D photonic bandgaps in silica/air structures," Elec. Lett., 31(22), 1941-1943 (1995).

[4] Broeng, J., Sondergaard, T., Barkou, S. E. et al., "Waveguidance by the photonic bandgap effect in optical fibres," J. of Opt. A: P. and Appl. Opt., 1(4) 477-482 (1999).

[5] Hoo, Y. L., Jin, W., Ho, H. L. et al., "Evanescent wave gas sensing using microstructure fibre," Lasers and Electro-Optics, 2001. Supplement, 8-9 suppl, (2001).

[6] Szpulak, M., Urbanczyk, W., Martynkien, T. et al., "Temperature sensitivity of photonic crystal holey fibers," Proc. SPIE 5028, 108-114 (2003).

[7] Jensen, J., Hoiby, P., Emiliyanov, G. et al., "Selective detection of antibodies in microstructured polymer optical fibers," Opt. Express, 13(15), 5883-5889 (2005).

[8] Polynkin, P., Polynkin, A., Peyghambarian, N. et al., "Evanescent field-based optical fiber sensing device for measuring the refractive index of liquids in microfluidic channels," Opt. Lett., 30(11), 1273-1275 (2005).

[9] Binu, S., Mahadevan Pillai, V. P., Pradeepkumar, V. et al., "Fibre optic glucose sensor," Mat. Sci. and Eng.: C, 29(1), 183-186 (2009).

[10] Degre, G., Joseph, P., Tabeling, P. et al., "Rheology of complex fluids by particle image velocimetry in microchannels," Appl. Phys. Lett., 89(2), 024104-3 (2006).

[11] Nielsen, K., Noordegraaf, D., Sorensen, T. et al., "Selective filling of photonic crystal fibres," J. of Opt. A: P. and Appl. Opt., 7(8), L13-L20 (2005).

[12] Rutowska, M., Horan, L., and Gunning, F. C. G., "Photonic Crystal Fibres: Selective filling delays," CLEO Europe - EQEC, 1 - 1, 2009.

[13] Birks, T., Bird, D., Hedley, T. et al., "Scaling laws and vector effects in bandgap-guiding fibres," Opt. Express, 12(1), 69-74 (2004).

[14] Antonopoulos, G., Benabid, F., Birks, T. A. et al., "Experimental demonstration of the frequency shift of bandgaps in photonic crystal fibers due to refractive index scaling," Opt. Express, 14(7), 3000-3006 (2006). 